\title{
Clinical Implication of Tinnitus Magnitude Index
}

\author{
Kyung Eun Lee ${ }^{1}$, Soo Hee Oh ${ }^{1,2}$, Junghwa Bahng ${ }^{1,2}$ \\ 'Department of Audiology and Speech-Langauge Pathology, Hallym University of Graduate Studies, Seoul, Korea \\ ${ }^{2}$ HUGS Center for Hearing and Speech Research, Seoul, Korea
}

\author{
이명규모지수(Tinnitus Magnitude Index)의 유용성 연구 \\ 이경은 $\cdot$ 오수희 ${ }^{1,2} \cdot$ 방정화 ${ }^{1,2}$ \\ 한림국제대학원대학교 청각언어치료학과', 한림청각언어연구소 ${ }^{2}$
}

\begin{abstract}
Purpose: Tinnitus magnitude index (TMI) is the questionnaire to evaluate the intensity of the tinnitus perception on the cognitive, behavioral, and emotional reaction to tinnitus. Whether TMI has a possible clinical implication as the Korean adaptation, the present study confirmed the correlations between the Korean adaptation of the TMI questionnaire and tinnitus handicap questionnaire (THQ), and TMI and psychoacoustic loudness from the tinnitogram. Methods: Although a total of 265 participants were recruited, we selected only 100 subjects who had tinnitus at the time of the psychoacoustic test of tinnitus and completed all the questions of THQ and TMI. The subjective loudness of the psychoacoustic test was compared to each of the TMI questions, and for the validation of the Korean adaptation of TMI, THQ was measured. Results: There was no significant relationship between each TMl questionnaire and the loudness from the tinnitogram. Regarding the correlation between each question of THQ response and each question of TMI, there was a significant moderate relationship. Conclusion: Taken together, there was no relationship between subjective loudness and the intensity of tinnitus perception of cognitive, behavioral, and emotional reaction to tinnitus, while TMI could assess the stress and pain from tinnitus. TMI is a concise, quick, and simple questionnaire, so it would be useful for evaluating the reaction of tinnitus perception in the clinical setting.
\end{abstract}

Key Words: Tinnitus, Tinnitogram, Tinnitus magnitude index, Tinnitus handicap questionnaire.

Received: June 6, 2019 / Revised: July 5, 2019 / Accepted: July 9, 2019

Correspondence: Junghwa Bahng, Department of Audiology and Speech-Langauge Pathology, Hallym University of Graduate Studies, 427 Yeoksam-ro, Gangnam-gu, Seoul 06197, Korea

Tel: +82-70-8680-6933 / Fax: +82-2-3453-6618 / E-mail: bahng.jh@gmail.com

\section{INTRODUCTION}

이명(tinnitus)은 외부의 특별한 소리 자극 없이 귀 또는 머리 속에서 느끼는 이상 음감(Jastreboff \& Hazell, 2004)을 말한 다. 대부분의 사람은 살면서 누구나 이명을 한 번 이상 경험한 다. 이명 증상은 일시적으로 발생했다가 사라지나(Snow, 2004), 일부의 경우 이명이 지속적으로 발생하여 어려움을 겪는다 (Axelsson \& Ringdahl, 1989; Hoffman \& Reed, 2004). 지속 적으로 발생하는 이명의 유병률은 이명을 평가하는 방법과 대 상군에 따라 차이가 있으나, Baguley et al.(2013)은 이명의 유 병률을 10 15\%로 보고하였다. 나라별 이명의 유병률 연구에서 는 미국 7.9\%(Shargorodsky et al., 2010), 영국 16.2\%(McCor-

(c) This is an Open Access article distributed under the terms of the Creative Commons Attribution Non-Commercial License (https://creativecommons.org/licenses/by-nc/4.0) which permits unrestricted non-commercial use, distribution, and reproduction in any medium, provided the original work is properly cited. mack et al., 2014), 일본 18.6\%(Michikawa et al., 2010)로 나타 났다. 국내 이명 연구에서도 10.5 20.3\%(Cho et al., 2010; Lee $\&$ Lee, 2007)의 유병률을 보고한 바 있고 이는 해마다 점점 증 가하는 추세이다(Kim et al., 2015).

임상에서 대상자의 이명을 정량화하기 위하여 심리음향검사 인 이명도 검사를 실시한다(Henry \& Meikle, 2000). 이명도 검사는 음량매칭을 통하여 대상자가 느끼고 있는 이명의 주파 수를 찾으며, 강도매치를 하여 이명의 크기를 찾게 된다. 그 다 음은 이 이명을 차폐하기 위한 최소강도 레벨을 찾고 $10 \mathrm{~dB}$ SL 수준에서 소음을 약 1 분간 제시하고 대상자에게 이명 소리 크 기의 변화를 다음과 같은 카테고리에서 어떻게 변하였는지 질 문한다. 첫째, 이명의 소리가 일시적으로 없어졌는지, 둘째, 이 명 소리의 크기가 작아졌는지, 셋째, 이명 소리의 크기가 변하 지 않았는지, 그리고 마지막으로 이명의 소리가 커졌는지 확인 한다. 이명도 검사의 결과에 따라 임상가는 대상자가 소리 발생 
기를 사용하였을 때 이명의 양상이 어떻게 될지 예측할 수 있 는 정보를 줄 수 있다. 만약 소음을 1분간 듣고 소리의 크기가 커졌다면 이명 차폐기 등을 사용할 경우 오히려 이명을 악화시 킬 우려가 있다(Yang \& Byun, 2016). 그러나 이러한 심리음향 적 이명의 측정은 검사자가 숙련되어야 하며, 측정 시 시간이 오래 걸리는 단점이 있다(Meikle et al., 2008). 또한 이명도 검 사의 방법은 문헌마다 병원마다 조금씩 다른 방법을 사용하고 있고 국제적으로도 표준화되지 않았다. 이러한 이명도의 검사 결과는 이명을 객관화하기 위한 이유로 쓰인다(Chang et al., 2014).

이명은 이명 증상뿐만 아니라 이명으로 인한 고통, 스트레스, 불안, 수면장애 등의 증세를 동반하고(Vesterager, 1994), 심리 및 정신적 어려움, 수면, 일, 집중력 등 일상생활에서의 불편함 을 초래하여 삶의 질 저하에 영향을 미칠 수 있다. 이러한 이명 으로 인한 고통은 이명 설문지로 평가를 하게 된다. 대표적으로 임상에서 쓰이는 이명장애지수(tinnitus handicap inventory, THI) (Newman et al., 1996)와 이명장애설문지(tinnitus handicap questionnaire, THQ) (Kuk et al., 1990)는 각각 25문항과 15 문항으로 이루어진 설문지이며 이명으로 인한 기능적이고 감 정적인 부정적 영향과 심각성을 묻는 질문으로 구성되어 있다. 이러한 설문지의 결과를 살펴보면 이명으로 인한 문제는 삶의 만족(Hallam et al., 1998), 우울(Newman et al., 1996), 화 (Wilson et al., 1991)와 관련이 있는 것으로 나타나 이명으로 인 한 부정적 영향을 평가하기 위해서는 설문지가 효과적인 측정 방법이 될 수 있다.

이명으로 인한 부정적인 영향과 어려움은 개인의 청각, 의학, 심리적 특성에 따라 다양하게 나타나며 이러한 차이를 만들어 내는 요인 중 하나가 이명의 강도이다(McCombe et al., 2001; Tyler \& Conrad-Armes, 1983; Tyler \& Stouffer, 1989). 지각 하는 이명강도가 크고 시끄러울수록 이명으로 인한 불편함이 더 심하며 이는 삶의 질 저하에 영향을 미칠 수 있다(Hiller \& Geobel, 2006). 이러한 이유로 이명강도는 이명으로 인한 불편 함을 판단하는 기준(Unterrainer et al., 2003)으로 활용된다. Schmitdt et al.(2014)은 이명의 지각(perception)과 이명으로 인 한 심리적 고통 반응(reaction)의 인과관계를 설명하기 위해 이 명규모지수(tinnitus magnitude index, TMI)를 개발하였다. 이 때 이명의 지각과 관련된 중요 변수 또는 예측 요인은 이명 규모 (magnitude)이며, 이명의 규모(magnitude)는 개인이 느끼는 이 명의 크기를 뜻한다. 또한 이명의 지각과 고통 반응의 인과관계 를 설명하는 과정에서 기능적 간섭(행동)과 자가 조절(인지)의 두 요인은 이명강도의 지각과 심리적 고통 반응 사이의 조정 변 수로 작용한다. 다시 말해 이명의 지각에서 기인된 행동 및 인 지 요소는 이명으로 인한 심리적 고통 반응에 영향을 미칠 수
있으며, 이명으로 인한 심리적 고통 반응을 중재 또는 조정할 수 도 있다. TMI는 예측 요인인 이명 규모(tinnitus magnitude), 조 정 요인인 기능적 간섭과 자가 조절(interference with functioning and self-control), 결과 요인인 심리적 불편함(psychological distress)으로 구성되는 3 가지 잠재요소와의 인과관계 이론을 배 경으로 한다. 이명에서 기인된 불편함(distress)에 대한 인지행동 조정모델(cognitive-behavioral mediation model) (Schmidt et al., 2014)은 TMI의 기본 모형으로, 이는 통증(pain)에 대한 인 지행동 조정모델(Rudy et al., 1988; Stouffer \& Tyler, 1990)과 감각적 지각 및 환경적 외부 요인과의 관계를 설명하는 적응수 준이론(adaptation level theory) (Helson, 1964)을 이명강도 의 지각과 연관시킨 이명에 대한 적응수준이론(Searchfield et al., 2012)을 근거로 개발되었다.

Schmidt et al.(2014)은 이명 크기(규모, magnitude)에 대한 지각만을 예측 변수로 활용하기 위하여 심리 및 감정적 요인과 같이 이명강도 요인 이외에 반응에 영향을 미칠 수 있는 혼재 요소를 최소화한 질문 항목으로 이명의 크기(how loud), 인지 (how aware), 심각성(how severe)으로 구성된 3가지의 기본 (prototype) TMI 항목을 개발하였다. 이는 각각 이명을 느끼는 반응에 대하여 인지, 행동 그리고 감정의 반응을 살펴보기 위 한 항목이다. Hwang \& Bahng(2018)은 Schmidt et al.(2014)의 질문을 토대로 한국어판 TMI를 개발하였다. 이명 규모에 대한 3 가지 질문 항목은 다음과 같다.

1) “지난 한 주 동안 이명은 얼마나 크게 들렸습니까?"

("Over the past week, how strong or loud was your tinnitus?")

2) "지난 한 주 동안 깨어있는 시간 중에 이명을 인지한 시간 은 몇 \% 정도입니까?”

("Over the past week, what percentage of your time awake were you consciously aware of your tinnitus?")

3) “지난 한 주 동안 이명이 얼마나 심각하게 느껴졌습니까?"

(“How severe has your tinnitus been over the past week?”) 특히 이명에 대한 주관적 지각을 파악하고자 하는 문항과 누구나 이해하기 쉬운 직관적 질문을 통해 위 세 영역을 정량 적으로 측정하고자 하였다. 임상에서 널리 쓰이고 있는 기존 설문지의 경우 25 개 내외의 많은 문항과 긴 설문 문장을 포함 하여 노인 또는 이해도가 다소 떨어지는 설문 대상자들에게 시 행이 번거로울 수 있다는 단점이 있다. 이러한 단점으로 인하여 $\mathrm{THI}$ 는 25 개의 문항 중 10 개의 항목을 채택하여 발표하였으며 높은 검사의 검사-재검사 신뢰성을 보였다(Mun et al., 2013). 그러나 TMI의 3 문항과 비교하여 보았을 때는 여전히 10 문항이 라는 많은 문항을 답해야 하는 어려움이 있다. 또한 기존 이명 설문지의 경우 이명에 대한 인지와 반응이 혼재되어 있는 설문 
등으로 인하여 임상가는 이명에 대한 대상자의 적절한 반응에 대하여 제대로 파악하지 못할 수도 있다. TMI는 이러한 단점 을 극복하여 짧은 문항으로 이명의 인지에 대한 반응만을 질문 으로 구성되어 있다. TMI와 임상에서 널리 쓰고 있는 THI를 비교한 Hwang \& Bahng(2018)과 Schmidt et al.(2014)의 연구 결과는 모두 TMI의 내적 상관도는 높은 것으로 나타났으며, 각 각의 THI 문항과 TMI 문항과의 상관관계는 유의한 중등도의 상관관계를 갖는 것으로 보고하였다.

본 연구의 목적은 첫째, 한국어 TMI (Hwang \& Bahng, 2018)를 사용하여 한국어 TMI 설문에서 파악한 이명 규모와 실제 이명도 검사에서 측정한 이명 크기(dB SL)와의 상관관계 를 파악하는 것이다. 이명도 검사에서 측정한 이명 크기와 $\mathrm{TMI}$ 의 주관적 이명 크기와의 상관성 분석을 통해 TMI 결과 를 이명의 심리적 고통 중재 시 임상적으로 활용하는 데 도움이 되고자 한다. 둘째, 한국어 $\mathrm{TMI}$ 와 이명 인지의 정량적 평가를 위해 개발된 한국어 이명장애설문(THQ Korea, THQ-K) (Jun et al., 2015)과의 상관관계를 분석하여 한국어 TMI 문항이 이 명 지각으로 인한 심리적 고통을 정량적으로 예측할 수 있는 도구로 활용될 수 있는지를 확인하고자 한다.

\section{MATERIALS AND METHODS}

\section{연구 대상}

본 연구는 2017년 4월부터 2018년 2월까지 이명을 주 증상으 로 서울 소재 이비인후과를 방문한 대상자 265명을 대상으로 하였다. 이 중 내원 당시 이명이 들려 이명도 검사가 가능하고 한국어 이명규모지수(TMI) 설문, 한국어 이명장애설문(THQ$\mathrm{K})$ 의 문항을 모두 대답한 100 명의 자료를 분석하였다. 대상자 의 연령은 만 14 세부터 85 세(평균연령 만 51세)까지였다. 정상 청력은 양이 순음청력평균 $(250 \sim 8,000 \mathrm{~Hz})$ 이 $20 \mathrm{~dB} \mathrm{HL}$ 이내 였으며, 난청 대상자의 청력은 경도 난청부터 고도 난청까지 다 양하였다. 또한 이명이 들리는 귀는 오른쪽 30 명, 왼쪽 34 명, 양 이 36 명이었다. 100 명 대상자의 평균 순음청력검사 결과 오른 쪽은 $23.3 \pm 15.5 \mathrm{~dB} \mathrm{HL}$, 왼쪽은 $25.9 \pm 20.6 \mathrm{~dB} \mathrm{HL}$ 이었다.

\section{이명도(Tinnitogram) 검사}

청력검사기(GSI 61, Grason-Stadler Inc., Eden Prairie, MN, USA)를 이용하여 이중벽 방음실에서 이명도 검사를 시행하였 다. 먼저 순음, 협대역잡음, 백색잡음을 제시하여 검사 대상자 의 이명과 가장 일치하는 소리 종류를 검사하였고, 선택한 소 리를 사용하여 이명과 일치하는 주파수를 검사하는 음조매칭 (pitch matching)을 시행하였다. 음조매칭한 주파수에서의 소리 강도를 $1 \mathrm{~dB}$ 간격으로 조정하면서 이명이 들리는 동측 귀를 기
준으로 이명과 음량을 비교하며 매치시키는 음량매칭(loudness matching) 검사를 시행하였다. 이명과 동일한 주파수의 역치 를 기준으로 음량매치한 이명강도를 $\mathrm{dB} \mathrm{SL}$ 값으로 환산하였고 양측 이명이 있는 경우에는 $\mathrm{dB} \mathrm{SL}$ 값이 큰 쪽 귀를 기준으로 하였다.

\section{설문지 검사}

각각의 설문은 청각검사 전 대기실에서 응답하였다. 설문의 목적을 설명하고 참여하기를 원하는 대상자만 설문지를 작성 하게 하였다. 질문이 이해가 안 되는 부분은 검사자에게 질문하 고 답변의 표기는 직접 하도록 하였다.

\section{이명규모지수}

주관적 이명 규모의 파악을 위해 한국어판 TMI 설문(Hwang \& Bahng, 2018)을 시행하였다. 총 3문항으로 구성되는 TMI 설 문의 내용과 각 문항의 답변은 다음과 같다. 먼저 "지난 한 주 동안 이명은 얼마나 크게 들렸습니까?” 문항에 대한 답은 0 (전 혀 크지 않았다)부터 10 (매우 크게 들렸다)까지 1 단위로 커지며 총 11 개(0 10)의 숫자 중 지각되는 이명의 크기 단계를 선택하 도록 하였다. 두 번째 "지난 한 주 동안 깨어있는 시간 중에 이 명을 인지한 시간은 몇 \% 정도입니까?” 문항은 0 (전혀 인지하 지 않았다)부터 100 (항상 인지했다)까지 10 단위로 커지는 총 11 개(0 100)의 퍼센트 단계를 이용하여, 깨어있는 시간 중 이명 을 인지하는 시간을 선택하도록 하였다. 세 번째 "지난 한 주 동 안 이명이 얼마나 심각하게 느껴졌습니까?” 문항은 응답자가 이 명을 얼마나 심하게 느꼈는지 $100 \mathrm{~mm}$ 줄 위에 해당하는 정도 를 체크하는 것으로, 점수는 0 (전혀 심각하지 않았다)부터 100 $\mathrm{mm}$ (매우 심각하다고 느꼈다)까지 선 위에 체크한 지점을 $\mathrm{mm}$ 로 측정하였다. TMI 설문의 평균 작성 시간은 2 분 내외였다.

\section{이명장애설문}

한국어판 이명장애설문(THQ-K) (Jun et al., 2015)은 이명 으로 인한 심리적 장애 정도 파악을 목적으로 청력, 생활 습관, 건강 및 감정 상태에 대한 이명의 잠재적 영향을 설명하는 총 27개 설문 문항으로 구성되며(Appendix), 각 문항은 다음 3가 지 구성요소(Factor 1-3)를 포함한다.

각 문항에 대한 답변은 '전혀 그렇지 않다'는 0점, '매우 그렇 다는 100점으로, 그 중간은 설문 대상자가 느끼는 대로 주관적 으로 결정하여 숫자를 기입하도록 하였다. 각 구성요소의 점수 계산법은 Figure 1 (Widex, 2019)과 같다.

\section{통계 분석}

통계 분석은 IBM SPSS statistics 21 (IBM Corp., Armonk, 
NY, USA)을 사용하여 Spearman's rank-order correlation을 시행하였다. 첫째, 한국어 TMI와 이명도의 음량매치검사에서 구한 이명강도와의 상관관계를 분석하고, 둘째, 한국어 TMI와 $\mathrm{THQ}-\mathrm{K}$ 의 3개 구성요소 간의 상관관계를 분석하고, 셋째, 한 국어 TMI와 THQ-K의 27개 문항별 상관관계를 분석하였다.

\section{RESULTS}

\section{한국어 TMI의 이명강도와 이명도 음량매치검사의 이명강도와의 상관관계}

이명의 음조매칭검사 결과 이명 소리의 종류는 협대역잡음 (narrowband noise) 47귀, 순음(pure tone) 82귀로 나타났으며, 이명 주파수는 $8 \mathrm{KHz}$ 가 34귀로 가장 많았다(Table 1). 음량매

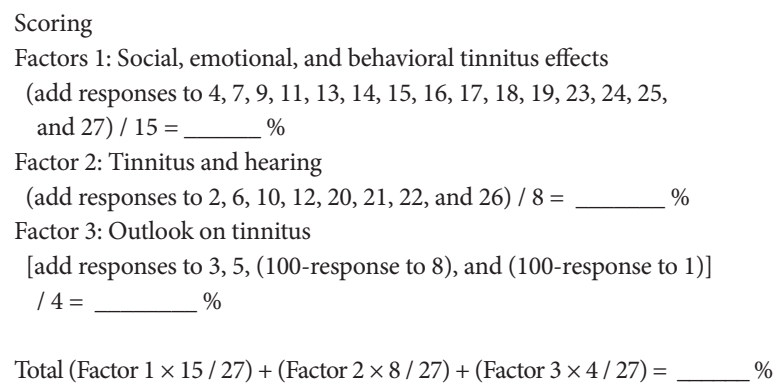

Figure 1. Tinnitus handicap questionnaire Scoring.

Table 1. Tinnitus characteristics and number of ears

\begin{tabular}{cc}
\hline Tinnitus characteristics & Number of ears \\
\hline Type & 47 \\
NBN & 82 \\
Pure tone & \\
Frequency $(\mathrm{kHz})$ & 6 \\
0.125 & 6 \\
0.25 & 11 \\
0.5 & 5 \\
0.75 & 17 \\
1 & 1 \\
1.5 & 16 \\
2 & 6 \\
3 & 14 \\
4 & 11 \\
6 & 34 \\
8 & 2 \\
12 &
\end{tabular}

NBN: narrow band noise
치검사에서 나타난 이명강도의 범위는 $-24 \mathrm{~dB}$ SL에서 $30 \mathrm{~dB}$ $\mathrm{SL}$ 이었고 평균은 $4.84 \pm 10.85 \mathrm{~dB}$ SL이었다. 양이 모두 이명 이 있을 경우 큰 값을 기준으로 하였다(Table 2).

$\mathrm{TMI}$ 의 3개 설문 문항 결과와 이명도 검사상 나타난 이명크 기와의 상관관계(Spearman's rank-order correlation)를 분석 한 결과, 이명 크기를 묻는 1번 질문과 이명에 대한 지각을 묻 는 2 번 질문, 마지막으로 이명의 심각함을 묻는 3번 질문 모두 이명도 검사의 음량매칭검사에서 나타난 이명강도와 유의하지 않은 낮은 상관관계를 보였다(Table 3). 이는 이명 크기, 이명 인 지, 이명의 심각함에 대한 주관적 TMI 설문 결과는 이명도 검 사상의 이명강도와 서로 관련이 없음을 보여준다.

\section{한국어 $\mathrm{TM}$ 와 $\mathrm{THQ}-\mathrm{K}$ 의 하위 구성요소 간의 상관관계}

한국어 TMI와 THQ-K 설문의 3개의 하위 구성요소(Factor 1-3)의 평균 점수는 Table 4와 같으며 Table 5는 TMI 3 개 점수 와 THQ-K 3개 하위 구성요소(Factor 1-3) 점수 및 총 점수 평 균 간의 상관관계를 분석한 결과이다.

$\mathrm{TMI}$ 의 각 항목별 점수와 $\mathrm{THQ}$ 3개 하위요소의 총합 평균의 상관관계를 분석했을 때 이명 크기에 대한 지각과 관계된 $\mathrm{TMI}_{1}$ 점수와 이명의 심각함과 관련된 $\mathrm{TMI}_{3}$ 점수는 중등도의 상관관계 $\left(\mathrm{TMI}_{1} ; \mathrm{rs}=0.636, \mathrm{TMI}_{2} ; \mathrm{rs}=0.669, p<0.001\right)$ 를 보 였으나 이명의 지각과 관련된 $\mathrm{TMI}_{2}$ 점수는 다른 두 문항에 비 해 $\mathrm{THQ}$ 의 3 영역의 하위요소 총합의 평균과 다소 낮은 상관관 계(rs $=0.294, p<0.001)$ 를 보였다. TMI 각 3 문항의 점수와 $\mathrm{THQ} 3$ 개 하위 구성요소와의 상관관계를 각각 분석한 결과 Factor 1과 Factor 2는 경도에서 중등도의 상관관계를 보였으 나, $\mathrm{TMI}_{2}$ 항목을 제외한 $\mathrm{TMI}_{1}$ 과 $\mathrm{TMI}_{3}$ 은 Factor 1과 Factor 2 에서 중등도의 상관관계를 보였다. 특히 정서적, 사회적, 이명의 신체적 영향을 나타내는 $\mathrm{THQ}$ Factor 1 과 $\mathrm{TMI}_{1}$ 과 $\mathrm{TMI}_{3}$ 점수 는 다른 요소보다 높은 상관관계를 보여 $\left(\mathrm{TMI}_{1} ; \mathrm{rs}=0.611\right.$, $\left.\mathrm{TMI}_{3} ; \mathrm{rs}=0.689, p<0.001\right)$ 이명 크기와 심각함에 대한 주관 적 지각이 이명으로 인한 정서적, 사회적, 이명의 신체적 영향 과 긍정적 상관관계에 있음을 알 수 있었다.

Table 3. Spearman's rank-order correlation between TMI and tinnitus loudness

\begin{tabular}{cccc}
\hline Tinnitogram & $\mathrm{TMI}_{1}$ & $\mathrm{TMI}_{2}$ & $\mathrm{TMI}_{3}$ \\
\hline Loudness of tinnitus & 0.120 & -0.034 & -0.027 \\
\hline
\end{tabular}

TMI: tinnitus magnitude index, $\mathrm{TMI}_{1}$ : TMI 1st question, $\mathrm{TMI}_{2}$ : TMI 2nd question, $\mathrm{TMI}_{3}$ : TMI 3rd question

Table 2. Distribution of subjective tinnitus loudness from tinnitogram

\begin{tabular}{ccccccccccc}
\hline Tinnitus loudness range (dB SL) & $<-15$ & $-15--10$ & $-9--5$ & $-4-0$ & $0-5$ & $6-10$ & $11-15$ & $16-20$ & $21-25$ & $\geq 25$ \\
\hline $\mathrm{n}$ & 4 & 6 & 2 & 13 & 20 & 24 & 21 & 5 & 3 & 5 \\
\hline
\end{tabular}

If the subject has tinnitus both sides, louder tinnitus level was selected 
Table 4. Average scores and SD of TMI and THQ

\begin{tabular}{|c|c|c|c|c|c|c|c|}
\hline \multirow{2}{*}{ Score } & \multicolumn{3}{|c|}{ TMI } & \multicolumn{4}{|c|}{ THQ } \\
\hline & $\mathrm{TMI}_{1}$ & $\mathrm{TMI}_{2}$ & $\mathrm{TMI}_{3}$ & Factor 1 & Factor 2 & Factor 3 & Total \\
\hline Average \pm SD & $6.1 \pm 2.4$ & $71.4 \pm 27.5$ & $61.7 \pm 26.3$ & $42.1 \pm 25.1$ & $33.9 \pm 24.9$ & $41.9 \pm 12.5$ & $39.6 \pm 20.5$ \\
\hline \multicolumn{8}{|c|}{$\begin{array}{l}\text { SD: standard deviation, TMI: tinnitus magnitude index, THQ: tinnitus handicap questionnaire, } \mathrm{TMI}_{1} \text { : TMI 1st question, TMI: TMI 2n } \\
\text { question, } \mathrm{TMI}_{3} \text { : TMI 3rd question }\end{array}$} \\
\hline TMI & THQ F & & THQ F2 & & THQ F3 & & otal \\
\hline $\mathrm{TMI}_{1}$ & 0.661 & & $0.574^{\dagger}$ & & 0.089 & & \\
\hline $\mathrm{TMI}_{2}$ & 0.299 & & $0.256^{*}$ & & -0.039 & & \\
\hline $\mathrm{TMI}_{3}$ & 0.689 & & $0.584^{\dagger}$ & & -0.128 & & \\
\hline
\end{tabular}

${ }^{*} p<0.1,{ }^{\dagger} p<0.05$. TMI: tinnitus magnitude index, THQ: tinnitus handicap questionnaire, THQ F1: physical, emotional, and social consequences of tinnitus, THQ F2: hearing ability of the patient, THQ F3: patients' view of tinnitus, THQ total: THQ-summation, TMI : TMI 1st question, $\mathrm{TMI}_{2}$ : TMI 2nd question, $\mathrm{TMI}_{3}$ : TMI 3rd question

\section{한국어 $\mathrm{TMl}$ 와 $\mathrm{THQ}$ 의 27 개 문항별 상관관계}

Table 6은 한국어 TMI와 THQ 27개 항목 점수 간의 상관계 수를 보여준다(rs = 0.053 0.694). $\mathrm{TMI}_{1}$ 과 $\mathrm{TMI}_{3}$ 모두 THQ 14 번 문항(나는 이명 때문에 안정을 할 수 없다)과 가장 높은 상 관관계 $\left(\mathrm{TMI}_{1} ; \mathrm{rs}=0.611, \mathrm{TMI}_{3} ; \mathrm{rs}=0.694\right)$ 를 보였다. $\mathrm{TMI}_{1}$ 과 $\mathrm{TMI}_{3}$ 과의 상관관계가 중등도 이상의 비교적 높은 상관관계를 보인 THQ 질문은 14 17번 문항과 27번 문항으로 모두 정서적, 사회적, 이명의 신체적 영향에 관한 하위요소(Factor 1)와 관련 된 항목이었다.

\section{DISCUSSIONS}

본 연구는 TMI가 이명으로 인한 심리적 고통의 예측, 중재 에 활용될 수 있는지 알아보고자 $\mathrm{TMI}$ 를 사용한 이명 크기, 인 지, 심각성에 대한 자가 설문 결과를 이명도 검사에서 측정한 이명강도와의 상관관계를 파악하고 TMI 설문 문항과 한국어 $\mathrm{THQ}$ 설문 문항과의 상관관계를 분석하였다.

첫째, TMI에서 측정한 이명 규모(이명 크기, 이명 인지, 이명 으로 인한 심각함의 정도)에 대한 주관적 지각은 이명도 검사 에서 측정한 이명의 심리음향적 음량 크기와 매우 낮은 상관관 계를 보였다. 이는 설문에서 응답한 이명에 대한 주관적 지각과 심리음향적 이명 크기 간 관련성이 없으며, 주관적 이명 크기가 크거나 작다고 해서 심리음향학적으로 측정한 이명 크기가 관련 성 있게 커지거나 작아지지 않음을 보여준다. 다시 말해, 이명이 얼마나 크게 들리는지에 대한 기준과 지각은 개인 차가 있으며 이명도 검사에서 측정한 이명의 크기(dB SL)와 이명 크기에 대 한 지각이 반드시 일치하지 않을 수 있다. Kuk et al.(1990)은 이 명도 검사에서의 이명 크기와 설문지로 측정한 이명으로 인한 불편함 사이에 높은 상관관계가 있음을 보고하였으나, 또 다른 몇몇 연구에서는 이명도 검사에서의 이명의 크기와 이명으로 인
Table 6. Spearman's ranked-order correlation between each TMI question and each $T H Q$ question

\begin{tabular}{|c|c|c|c|}
\hline THQ & $\mathrm{TMI}_{1}$ & $\mathrm{TMI}_{2}$ & $\mathrm{TMI}_{3}$ \\
\hline$\overline{\text { THQ } 1}$ & $0.539^{\dagger}$ & $0.328^{\dagger}$ & $0.669^{\dagger}$ \\
\hline THQ 2 & $0.346^{\dagger}$ & 0.112 & $0.415^{\dagger}$ \\
\hline THQ 3 & $0.551^{\dagger}$ & $0.208^{\dagger}$ & $0.464^{\dagger}$ \\
\hline THQ 4 & $0.446^{\dagger}$ & $0.249^{*}$ & $0.351^{\dagger}$ \\
\hline THQ 5 & $0.546^{\dagger}$ & $0.207^{\dagger}$ & $0.398^{\dagger}$ \\
\hline THQ 6 & $0.502^{\dagger}$ & $0.336^{\dagger}$ & $0.392^{\dagger}$ \\
\hline THQ 7 & $0.572^{\dagger}$ & $0.271^{\dagger}$ & $0.458^{\dagger}$ \\
\hline THQ 8 & $0.397^{\dagger}$ & 0.174 & $0.411^{\dagger}$ \\
\hline THQ 9 & $0.458^{\dagger}$ & 0.101 & $0.467^{\dagger}$ \\
\hline THQ 10 & $0.479^{\dagger}$ & 0.105 & $0.388^{\dagger}$ \\
\hline THQ 11 & $0.483^{\dagger}$ & 0.111 & $0.531^{\dagger}$ \\
\hline THQ 12 & $0.299^{\dagger}$ & 0.053 & $0.401^{\dagger}$ \\
\hline THQ 13 & $0.396^{\dagger}$ & $0.308^{\dagger}$ & $0.557^{\dagger}$ \\
\hline THQ 14 & $0.611^{\dagger}$ & $0.314^{\dagger}$ & $0.694^{\dagger}$ \\
\hline THQ 15 & $0.514^{\dagger}$ & $0.318^{\dagger}$ & $0.682^{\dagger}$ \\
\hline THQ 16 & $0.622^{\dagger}$ & $0.226^{\dagger}$ & $0.636^{\dagger}$ \\
\hline THQ 17 & $0.587^{\dagger}$ & $0.274^{*}$ & $0.659^{\dagger}$ \\
\hline THQ 18 & $0.504^{\dagger}$ & $0.243^{*}$ & $0.673^{\dagger}$ \\
\hline THQ 19 & $0.374^{\dagger}$ & 0.192 & $0.510^{\dagger}$ \\
\hline THQ 20 & $0.535^{\dagger}$ & 0.200 & $0.476^{\dagger}$ \\
\hline THQ 21 & $0.351^{\dagger}$ & 0.188 & $0.332^{\dagger}$ \\
\hline THQ 22 & $0.341^{\dagger}$ & $0.238^{*}$ & $0.491^{\dagger}$ \\
\hline THQ 23 & $0.348^{\dagger}$ & 0.196 & $0.338^{\dagger}$ \\
\hline THQ 24 & $0.409^{\dagger}$ & $0.228^{*}$ & $0.601^{\dagger}$ \\
\hline THQ 25 & 0.074 & $0.262^{*}$ & 0.068 \\
\hline THQ 26 & $0.468^{\dagger}$ & $0.268^{*}$ & $0.484^{\dagger}$ \\
\hline THQ 27 & $0.504^{\dagger}$ & 0.097 & $0.550^{\dagger}$ \\
\hline
\end{tabular}

${ }^{*} p<0.1,{ }^{\dagger} p<0.05$. TMI: tinnitus magnitude index, THQ: tinnitus handicap questionnaire, $\mathrm{TMI}_{1}$ : TMI 1st question, $\mathrm{TMI}_{2}$ : TMI 2nd question, $\mathrm{TMI}_{3}$ : TMI 3rd question

한 핸디캡과의 상관관계가 높지 않다(Jastreboff \& Hazell, 2004; Nascimento et al., 2018; Ryu \& Bahng, 2011)고 보고하 여 이명의 주관적 지각, 불편함과 이명도 검사상의 이명 크기와 
의 상관관계는 연구마다 다른 견해를 보인 바 있다.

둘째, TMI와 THQ-K 점수와의 상관관계를 분석한 결과 $\mathrm{TMI} 1$ 번과 3 번 문항인 이명 크기와 이명의 심각함과 관련된 문 항은 THQ-K의 정서적, 사회적, 이명의 신체적 영향에 관한 요 소(Factor 1) 및 청력 및 의사소통에 관한 요소(Factor 2)와 중 등도의 상관관계를 보였다. 이 중 특히 정서적, 사회적, 이명의 신체적 영향에 관한 요소(Factor 1)와 관련된 문항과는 전반적 으로 높은 상관관계를 보여 TMI 1번과 3번 문항이 이명으로 인한 정서, 사회, 신체적 영향과 관련이 있음을 확인하였다. TMI와 THI (Newman et al., 1996)와의 상관관계를 분석한 선 행연구(Hwang \& Bahng, 2018)에서도 이와 비슷한 결과를 보 고한 바 있다. TMI는 THI의 이명에서 기인된 기능, 감정, 재앙 3 영역의 총합 평균점수와 중등도의 상관관계 $(\mathrm{r}=0.403 \sim 0.530)$ 를 보였으며 이 중 $\mathrm{TMI}_{1}$ 과 $\mathrm{TMI}_{3}$ 은 $\mathrm{THI}$ 의 기능 영역과 좀 더 높 은 상관관계를 보였다 $(\mathrm{r}=0.522 \sim 0.562)$.

이명과 관련된 설문검사의 유용성 측면에서 TMI (Schmidt et al., 2014)와 THQ (Kuk et al., 1990)의 차이점을 살펴보면 $\mathrm{TMI}$ 는 이명 규모에 대한 지각을 측정하고 $\mathrm{THQ}$ 는 청력, 생활 습관, 건강 및 감정 상태에 대한 이명의 잠재적 영향을 모두 측 정하는 데 그 목적이 있다. 또한 $\mathrm{TMI}$ 와 $\mathrm{THQ}$ 의 응답과 점수 산 출 방법에도 차이가 있는데, THQ 응답자는 각 항목별로 '전혀 그렇지 않다' 0 점, '매우 그렇다' 100 점 사이의 값을 주관적으로 결정하여 숫자를 기입하기 때문에 주관적 점수 결정 시 시간이 소요되거나 판단의 어려움을 겪을 수 있다. 이에 비해 TMI는 0 10점 또는 0 100점 사이의 총 11개 점수 중 하나를 선택하 거나 시각적 스케일로 제시된 0 100점 사이의 특정 지점을 표 시하도록 하여 응답자의 반응을 좀 더 편안하게 이끌어 낼 수 있다는 장점이 있다. 본 연구에서도 THQ-K 설문 시 문항별 점 수 표기에 대한 응답자들의 이해도가 낮아 설문 시행이 용이하 지 않았으며, 비교적 많은 THQ-K 27개 설문 문항에 대한 부담 과 점수 기입의 불편함으로 누락된 응답을 제출하는 경우도 많 았다. 이러한 이유로 총 265 명 중 100 명의 결과만을 본 연구에 서는 분석할 수 있었다. 이러한 답변 누락의 문제는 TMI 3번 설 문과 같이 시각적 스케일을 사용하여 위치를 표시하는 응답 형 태를 활용한다면 보다 유용한 설문검사가 될 것이라고 예상할 수 있다. 그러나 시각적 스케일을 이용할 경우 점수를 수치화하 기 위하여 자로 재야 하는 불편함이 존재하지만 이명재활의 효 과를 전후로 비교하는 데 있어서는 한눈으로 비교 가능하기 때 문에 효과적이라 할 수 있다.

이명에서 기인된 불편함(distress)에 대한 인지행동 조정모델 을 기반으로 개발된 TMI는 이명의 규모 지각 측정을 위한 3가 지 문항을 기반으로 이명으로 인한 정신적 고통, 스트레스와의 상호관계를 설명한다는 점에서 의미가 있으며 $\mathrm{THQ}-\mathrm{K}$ 와의 상
관관계 분석을 통해 본 연구에서도 이를 뒷받침하고 있다. 그러 나 구체적인 이명으로 인한 불편함, 예를 들어 이명으로 인한 수면장애, 이명으로 인한 사회적 활동의 방해, 삶의 즐거움에 끼치는 영향 등을 알 수는 없다. 이러한 문제를 구체적으로 파 악하기 위해서는 $\mathrm{THQ}$ 혹은 $\mathrm{THI}$ 와 같은 설문지를 활용하여야 할 것이다. 그러나 보청기를 통한 이명 완화의 효과, 혹은 이명 재활훈련 전후의 비교 등 전반적인 이명에 관한 문제를 빠르게 파악하기 위한 목적으로는 TMI를 유용하게 이용할 수 있다.

임상에서 보다 효과적으로 이용하기 위해서는 아래와 같은 후속 연구가 더 필요하다. Meikle et al.(2008)은 적은 문항의 설 문지와 설문 구간의 척도가 적을 경우 신뢰도가 확보되지 않을 수 있다는 단점을 지적하였다. 향후 한국어 TMI에 대한 검사재검사 신뢰도 연구를(Schmidt et al., 2014) 시행하여 한국어의 TMI 설문에 대한 검증이 보완되어야 하며, TMI를 통한 이명 규모의 지각이 이명으로 인한 정신적인 스트레스를 예측할 수 있는지 알아보기 위해 심리적 상태에 중점을 둔 상태불안척도 (state trait anxiety inventory) (Spielberger et al., 1970)나 벡 우울척도(Beck depression inventory) (Beck et al., 1996)와 같 은 설문지와의 상관관계를 분석하는 일도 필요할 것이다. 또한 $\mathrm{TMI}$ 가 이명 규모 측정과 이명으로 인한 심리적 고통을 예측하 고 조정할 수 있는 검사로 활용되기 위해서는 응답 척도와 기준 확립에 대한 연구가 지속되어야 하며 지각되는 이명의 크기와 실질적 이명 크기와의 관계를 규명하고 이를 설명하는 후속 연 구가 필요하다.

중심 단어 : 이명·이명도·이명장애지수·이명핸디캡설문지.

\section{Ethical Statement}

The entire procedures including recruitment of participants, testing, and data handling were approved by the Institutional Review Board of the Hallym University of Graduate Studies (HUGS 697128). All authors followed the procedures.

\section{Acknowledgments}

The authors thank to the participants.

\section{Declaration of Conflicting Interests}

There are no conflict interests.

\section{Funding}

N/A

\section{Author Contributions}

All authors contributed equally to this work. K.L., S.O., and J.B. wrote the manuscript. K.L. and J.B. designed the study, and K.L. performed experiments and collected the data. K.L. and J.B. analyzed the data and S.O. provided the critical revision. Also, J.B. reviewed the final revision.

\section{ORCID iDs}

Kyung Eun Lee

Junghwa Bahng https://orcid.org/0000-0003-3685-8669 https://orcid.org/0000-0002-5265-3586 


\section{REFERENCES}

Axelsson, A. \& Ringdahl, A. (1989). Tinnitus--a study of its prevalence and characteristics. British Journal of Audiology, 23(1), 53-62.

Baguley, D., McFerran, D., \& Hall, D. (2013). Tinnitus. Lancet, 382(9904), 1600-1607.

Beck, A. T., Steer, R. A., \& Brown, G. K. (1996). Manual for the Beck Depression Inventory-II. San Antonio, TX: Psychological Corporation.

Chang, J., Kim, T. S., Nam, E. C., Moon, I. S., Park, M. K., Park, S. N., et al.. (2014). Current trends in the assessment of patients with tinnitus and the proposal of tinnitus assessment in Korea. Korean Journal of Otorhinolaryngology-Head and Neck Surgery, 57(10), 671-686.

Cho, Y. S., Choi, S. H., Park, K. H., Park, H. J., Kim, J. W., Moon, I. J., et al. (2010). Prevalence of otolaryngologic diseases in South Korea: Data from the Korea national health and nutrition examination survey 2008. Clinical and Experimental Otorhinolaryngology, 3(4), 183-193.

Helson, H. (1964). Adaptation-Level Theory: An Experimental and Systematic Approach to Behavior. New York, NY: Harper and Row.

Henry, J. A. \& Meikle, M. B. (2000). Psychoacoustic measures of tinnitus. Journal of the American Academy of Audiology, 11(3), 138-155.

Hiller, W. \& Goebel, G. (2006). Factors influencing tinnitus loudness and annoyance. Archives of Otolaryngology--Head and Neck Surgery, 132(12), 1323-1330.

Hoffman, H. J. \& Reed, G. W. (2004). Epidemiology of tinnitus. In Snow, J. B. Tinnitus: Theory and Management (pp. 16-41). Hamilton: BC Decker Inc.

Hwang, D. H. \& Bahng, J. (2018). A Korean adaptation of the tinnitus magnitude index: Validity and reliability. Audiology and Speech Research, 14(2), 90-99.

Jastreboff, P. J. \& Hazell, J. W. P. (2004). Tinnitus Retraining Therapy: Implementing the Neurophysiological Model. New York, NY: Cambridge University Press.

Jun, H. J., Yoo, I. W., Hwang, S. J., \& Hwang, S. Y. (2015). Validation of a Korean version of the tinnitus handicap questionnaire. Clinical and Experimental Otorhinolaryngology, 8(3), 198-201.

Kim, H. J., Lee, H. J., An, S. Y., Sim, S., Park, B., Kim, S. W., et al. (2015). Analysis of the prevalence and associated risk factors of tinnitus in adults. PLoS One, 10(5), e0127578.

Kuk, F. K., Tyler, R. S., Russell, D., \& Jordan, H. (1990). The psychometric properties of a tinnitus handicap questionnaire. Ear and Hearing, 11(6), 434-445.

Lee, S. J. \& Lee, S. K. (2007). Relationships of tinnitus to frequency and hearing loss in elderly patients. Korean Journal of Otorhinolaryngology-Head and Neck Surgery, 50(10), 869-875.

Lee, W. C., Choi, B. C., Oh, M. H., Park, C. Y., Kim, H., \& Koo, J. W. (1999). Prevalence of tinnitus and hearing thresholds of a non-noise-exposed population with and without tinnitus. Annals of Occupational and Environmental Medicine, 11(3), 323-331.

McCombe, A., Baguley, D., Coles, R., McKenna, L., McKinney, C., \& Windle-Taylor, P.; British Association of Otolaryngologists, Head and Neck Surgeons. (2001). Guidelines for the grading of tinnitus severity: The results of a working group commissioned by the British Association of Otolaryngologists, Head and Neck Surgeons, 1999. Clinical Otolaryngology and Allied Sciences, 26(5), 388-393.

McCormack, A., Edmondson-Jones, M., Fortnum, H., Dawes, P., Middleton, H., Munro, K. J., et al. (2014). The prevalence of tinnitus and the relationship with neuroticism in a middle-aged UK population. Jour- nal of Psychosomatic Research, 76(1), 56-60.

Meikle, M. B., Stewart, B. J., Griest, S. E., \& Henry, J. A. (2008). Tinnitus outcomes assessment. Trends in Amplification, 12(3), 223-235.

Michikawa, T., Nishiwaki, Y., Kikuchi, Y., Saito, H., Mizutari, K., Okamoto, M., et al. (2010). Prevalence and factors associated with tinnitus: A community-based study of Japanese elders. Journal of Epidemiology, 20(4), 271-276.

Mun, M. J., Goh, E. K., Oh, S. J., Kwon, H. K., Kong, S. K., \& Lee, I. W. (2013). Development of simplified version of Korean-type tinnitus handicap inventory. Korean Journal of Otorhinolaryngology-Head and Neck Surgery, 56(3), 138-143.

Nascimento, I. da P., Almeida, A. A., Diniz, J. J., Martins, M. L., Cruz deFreitas, T. M. M. W., Diniz da Rosa, M. R. (2018). Tinnitus evaluation: Relation among pitch matching and loudness, visual analog scale and tinnitus handicap inventory. Brazilian Journal of Otorhinolaryngology, pii: S1808-8694(18)30338-0.

Newman, C. W., Jacobson, G. P., \& Spitzer, J. B. (1996). Development of the tinnitus handicap inventory. Archives of Otorhinolaryngology-Head and Neck Surgery, 122(2), 143-148.

Rudy, T. E., Kerns, R. D., \& Turk, D. C. (1988). Chronic pain and depression: Toward a cognitive-behavioral mediation model. Pain, 35(2), 129140.

Ryu, S. J. \& Bahng, J. (2011). Analysis of factors affecting Tinnitus Handicap Inventory (THI). Audiology and Speech Research, 7(2), 164-178.

Schmidt, C. J., Kerns, R. D., Griest, S., Theodoroff, S. M., Pietrzak, R. H., \& Henry, J. A. (2014). Toward development of a Tinnitus Magnitude Index. Ear and Hearing, 35(4), 476-484.

Searchfield, G. D., Kobayashi, K., \& Sanders, M. (2012). An adaptation level theory of tinnitus audibility. Frontiers in Systems Neuroscience, 6, 46.

Shargorodsky, J., Curhan, G. C., \& Farwell, W. R. (2010). Prevalence and characteristics of tinnitus among US adults. The American Journal of Medicine, 123(8), 711-718.

Snow, J. B. (2004). Tinnitus: Theory and Management (pp. 1-39). Hamilton: BC Decker Inc.

Spielberger, C. D., Gorsuch, R. L., \& Lushene, R. E. (1970). The State-Trait Anxiety Inventory (Test Manual). Palo Alto, CA: Consulting Psychologists Press.

Stouffer, J. L. \& Tyler, R. S. (1990). Characterization of tinnitus by tinnitus patients. The Journal of Speech and Hearing Disorders, 55(3), 439-453.

Tyler, R. S. \& Conrad-Armes, D. (1983). The determination of tinnitus loudness considering the effects of recruitment. Journal of Speech and Hearing Research, 26(1), 59-72.

Tyler, R. S. \& Stouffer, J. L. (1989). A review of tinnitus loudness. Hearing Journal, 42, 52-57.

Unterrainer, J., Greimel, K. V., Leibetseder, M., \& Koller, T. (2003). Experiencing tinnitus: Which factors are important for perceived severity of the symptom? The International Tinnitus Journal, 9(2), 130-133.

Vesterager, V. (1994). Combined psychological and prosthetic management of tinnitus: A cross-sectional study of patients with severe tinnitus. British Journal of Audiology, 28(1), 1-11.

Widex. (2019, May). Tinnitus Handicap Questionnaire (THQ). Widex. Retrieved from http://webfiles.widex.com/WebFiles/9\%20502\%203106\% 20001\%2001.pdf.

Yang, C. W. \& Byun, J. Y. (2016). Tinnitus assessment. Hanyang Medical Reviews, 36(2), 109-112. 


\section{Korean Version of Tinnitus Handicap Questionnaire}

1. 나는 이명 때문에 삶을 즐길 수 없다.

2. 나의 이명은 매년 나빠진다.

3. 이명 때문에 소리가 어디에서 나는지 분간하기 어렵다.

4. 이명 때문에 다른 사람들과의 대화를 따라갈 수가 없다.

5. 이명 때문에 소음이 있는 곳을 피한다.

6. 이명 때문에 소음이 있는 곳에서 다른 사람과 대화하기가 힘들다.

7. 이명 때문에 사회 활동이 어렵다.

8. 다른 사람들은 이명이 얼마나 고통스러운지를 모른다.

9. 이명 때문에 집중할 수가 없다.

10. 이명 때문에 가족들과의 관계에 문제가 생긴다.

11. 나는 이명 때문에 기분이 우울해진다.

12. 나는 이명이 어떠한지를 다른 사람에게 설명하기 어렵다.

13. 이명은 나에게 스트레스를 준다.

14. 나는 이명 때문에 안정을 할 수 없다.

15. 나는 이명 때문에 불평이 많다.

16. 이명 때문에 밤에 잠들기가 어렵다.

17. 이명은 나를 지치게 한다.

18. 이명은 나를 불안하게 한다.

19. 나는 이명 때문에 내 전반적인 건강이 나쁘다고 생각한다.

20. 이명은 나의 대인관계에 영향을 미친다.

21. 나는 이명 때문에 말을 이해하는 능력이 떨어진다.

22. 나는 이명 때문에 짜증이 난다.

23. 나는 이명 때문에 텔레비전에서 나오는 말을 이해하기 어렵다.

24. 나는 이명 때문에 걱정이 많다.

25. 나는 나의 이명에 대해 낙관적인 생각을 가지고 있다고 생각한다.

26. 이명에 대해서 나의 친구들은 나를 돕는다.

27. 나는 이명 때문에 자주 좌절한다. 